\title{
NANOCIÊNCIA E NANOTECNOLOGIA COMO TEMÁTICAS PARA DISCUSSÃO DE CIÊNCIA, TECNOLOGIA, SOCIEDADE E AMBIENTE*
}

\section{Nanoscience and Nanotechnology as topical themes for a discussion on science, technology, society and environment}

\author{
Rodrigo Siqueira-Batista ${ }^{1}$ \\ Luciana Maria-Da-Silva ${ }^{2}$ \\ Roberto Rômulo de Medeiros Souza ${ }^{3}$ \\ Henrique Jannuzzelli Pires-Do-Prado ${ }^{4}$
}

\author{
Cláudio Aprígio da Silva ${ }^{5}$ \\ Giselle Rôças ${ }^{6}$ \\ Alexandre Lopes de Oliveira ${ }^{7}$ \\ José Abdalla Helayël-Neto ${ }^{8}$
}

Resumo: A educação científica e tecnológica tem sido intensamente discutida hodiernamente, tendo em vista o compromisso último das diferentes instituições de ensino: formar cidadãos capazes de entender o mundo no qual estão inseridos. Com efeito, a apreciação de temas da atualidade se constitui em abordagem bastante salutar para motivar as discussões sobre tais temáticas em sala de aula. Neste domínio se inserem a nanociência e a nanotecnologia, saberes que podem ser abordados de forma interdisciplinar, envolvendo as ciências exatas e da natureza de forma articulada às ciências humanas e sociais. A proposição de eixos temáticos para o emprego da nanociência e da nanotecnologia como mote para o ensino de ciências, construídos a partir da revisão da literatura sob um enfoque ciência-tecnologia-sociedade-ambiente (CTSA) é o escopo do presente artigo.

Palavras-chave: Ciência, Tecnologia, Sociedade e ambiente (CTSA). Educação. Nanociência. Nanotecnologia.

\begin{abstract}
Scientific and technological education has been, in present times, fairly-well discussed, with focus on the ultimate commitment of the various teaching institutions: to promote the formation of citizens who are able to understand the world where they live in. Actually, the choice of contemporary topics is by itself a very strategic approach to motivate discussions on such matters in the classroom. Nanoscience and nanotechnology come up very properly in such a domain, which can be treated in an interdisciplinary way, by joining exact and natural sciences on the one hand, and human and social sciences on the other hand. The proposal of thematic directions to approach nanoscience and nanotechnology as a motto for science teaching, set up from reviewing the literature under the optics of Science-Technology-Society-Environment (STSE), is the main goal of the present paper.
\end{abstract}

Keywords: Science, Technology, Society and environment (STSE). Education. Nanoscience. Nanotechnology.

\footnotetext{
* Este ensaio é uma retomada de Siqueira-Batista et al. (2009).

${ }^{1}$ Bacharel em Medicina e em Filosofia, doutor em Ciências. Docente, Departamento de Medicina e

Enfermagem, Universidade Federal de Viçosa. Viçosa, MG, Brasil. < rsbatista@ufv.br>

2,5 Licenciados em Física, Instituto Federal de Educação, Ciência e Tecnologia do Rio de Janeiro (IFRJ).

Nilópolis, RJ.<lumster@bol.com.br>; <claudinho.dtbr7@hotmail.com>

3,4 Graduandos em Medicina, UNIFESO. Teresópolis, RJ. < robertoromulo@gmail.com>; <monfas@msn.com>

${ }^{6}$ Bacharel em Biologia, doutora em Ecologia. Docente, IFRJ. Nilópolis, RJ. <grocas@gmail.com>

${ }^{7}$ Bacharel e doutor em Física. Docente, IFRJ. Nilópolis, RJ. <alexandre.l.deoliveira@gmail.com>

${ }^{8}$ Bacharel e phD em Física. Centro Brasileiro de Pesquisas Físicas. Rio de Janeiro, RJ. < helayel@cbpf.br>

${ }^{1}$ Universidade Federal de Viçosa, Departamento de Medicina e Enfermagem

Avenida Peter Henry Rolfs, s/n

Campus Universitário - Viçosa, MG

36.570-000

479

Ciência $\mathfrak{E}^{2}$ Educação, v. 16, n. 2, p. 479-490, 2010
} 
Siqueira-Batista, R. et al.

\section{Introdução}

A 'natureza' das ciências da natureza, suas relações com a tecnologia - quiçá pensadas em termos de tecnociência - e seu papel na educação para a vida em sociedade são temas recorrentes na agenda do pensamento, no Ocidente, ao longo dos últimos séculos. De fato, podem se recuperar as indagações propostas por Jean-Jacques Rousseau - e rediscutidas por Boaventura de Sousa Santos (2003) - no seu célebre Discurso sobre as ciências e as artes (ROUSSEAU, 1971):

Há alguma relação entre a ciência e a virtude? Há alguma razão de peso para substituirmos o conhecimento vulgar que temos da natureza e da vida e que partilhamos com os homens e mulheres da nossa sociedade pelo conhecimento científico produzido por poucos e inacessível à maioria? Contribuirá a ciência para diminuir o fosso crescente na sociedade entre o que se é e o que se aparenta ser, o saber dizer e o saber fazer, entre teoria e prática? (SANTOS, 2003, p. 16)

O desconforto provocado, ainda hoje, pelas questões de Rousseau mantém evidente ressonância no terreno pedagógico, na medida em que se discute a relevância da educação científica e tecnológica (ECT) para fomentar, no estudante, a capacidade de entendimento acerca do mundo no qual se vive. A aposta - feita por cientistas e educadores - é de que oferecer a possibilidade, aos estudantes, de adquirir conhecimentos acerca das ciências da natureza e da tecnologia pode, de fato, ser um elemento decisivo para o posicionamento crítico e responsável do indivíduo, em relação aos díspares problemas existentes na sociedade (MORTIMER, 2002; CHASSOT, 2006).

O alcance deste objetivo dependerá, evidentemente, da adoção de uma ECT críticapautada não somente na mera apresentação dos conteúdos programáticos das diferentes disciplinas, mas, sobretudo, na contextualização dos aspectos filosóficos, históricos, políticos, sociais e econômicos correlatos (BOMFIM, 2009) - a qual concorrerá para a formação de aprendizes e de professores reflexivos, domínio relevante para que os processos de inclusão social possam ocorrer (CHASSOT, 2006; MOREIRA, 2006). Para isto, pode-se lançar mão, nos processos de construção curricular, da abordagem Ciência-Tecnologia-Sociedade-Ambiente (CTSA), aspecto que, apesar de contemplado nos Parâmetros Curriculares Nacionais e nas diferentes diretrizes e orientações curriculares, não se formaliza, amiúde, nas salas de aula, ao menos não de forma perene e contextualizada (AMORIM, 2001; FARIAS e CARVALHO, 2006; MUENCHEN e AULER, 2007). De fato, tem sido identificada considerável distância entre a prática e a teoria em relação à inserção do discurso de CTSA na ECT, em função de diferentes fatores, podendo ser citados, dentre eles, a defasagem entre a formação dos professores e as demandas de uma nova escola para uma sociedade em constante transformação (FOUREZ, 2003; TEIXEIRA, 2003; ALBUQUERQUE et al., 2009; SIQUEIRA-BATISTA e SIQUEIRA-BATISTA, 2009).

O desafio de construir uma ECT crítica passa, igualmente, pela elaboração e consecução de atividades didáticas interessantes para o educando, em uma perspectiva que alie: (1) o enfoque CTSA, (2) a interdisciplinaridade e (3) a complexidade (SANT'ANNA e MENEGO- 
Nanociência e nanotecnologia como temáticas ...

LLA, 2007; RÔÇAS, MONTEIRO e SIQUEIRA-BATISTA, 2008; BOMFIM e SIQUEIRABATISTA, 2009). Com efeito, tem se tornado uma necessidade buscar assuntos emergentes na contemporaneidade e que possam ser abordados sob uma perspectiva interdisciplinar, destacando-se, neste âmbito, a nanociência e a nanotecnologia (TEDESCO, SIMIONI e PRIMO, 2007; SIQUEIRA-BATISTA et al., 2008), campos que vêm contribuindo, de modo salutar, para o desenvolvimento do conhecimento, fazendo-se presentes em publicações científicas da atualidade (TOMA, 2005; GEWIN, 2009). Do ponto de vista conceitual, pode-se comentar que:

Os termos nanociências e nanotecnologias se referem, respectivamente, ao estudo e às aplicações tecnológicas de objetos e dispositivos que tenham ao menos uma de suas dimensões físicas menores que, ou da ordem de, algumas dezenas de nanômetros. Nano (do grego "anão") é um prefixo usado nas ciências para designar uma parte em um bilhão e, assim, um nanômetro $(1 \mathrm{~nm})$ corresponde a um bilionésimo de um metro [...] "Nano", portanto, é uma medida e não um objeto. (LÊDO, 2006, p. 1)

A relevância adquirida pela nanotecnologia tem sido bastante significativa, permitindo o desenvolvimento de novos métodos e técnicas, já com aplicabilidade em diferentes procedimentos industriais - produção de alimentos, cosméticos, fármacos, tecidos, dentre outros - de um modo extremamente singular:

A proposta central da nanotecnologia é assim a de montar, a partir da manipulação individual de átomos e moléculas, dispositivos moleculares milhares de vezes menores que um fio de cabelo, capazes de construir outros dispositivos e máquinas, encaixando moléculas uma a uma, com uma precisão e eficiência impressionantes, e a um custo reduzido já que a matéria-prima - os átomos - afinal existe em abundância na natureza... (OLIVEIRA, 2002, p. 209)

Conforme esta perspectiva, podem-se identificar múltiplas possibilidades de discussão sobre sua atualidade, as quais podem - e devem - constituir mote para atividades na área de ECT, no espaço da sala de aula, especialmente no contexto da abordagem de CTSA.

É nesse domínio que o presente artigo foi construído. Seu escopo é, pois, a retomada da delimitação preliminar de eixos temáticos relativos à nanotecnologia (SIQUEIRA-BATISTA et al., 2009) - em suas relações com distintas esferas da sociedade -, os quais poderão ser empregados como assuntos para a ECT crítica.

\section{Métodos}

O presente trabalho constitui uma investigação teórica, alicerçada na revisão de literatura, utilizando a Scientific Eletronic Library Online (SCIELO) e a U. S. National Library of Medi- 
Siqueira-Batista, R. et al.

cine (PUBMED) como base. Os descritores empregados na busca foram "nanotecnologia", no SCIELO, e "nanotechnology" + "education", no PUBMED. Para complementar a revisão, foram também consultados livros e capítulos de livro atinentes à nanociência e à nanotecnologia. Com base na leitura dos manuscritos obtidos foi possível construir uma síntese reflexiva sobre o tema, o que permitiu a proposição de quatro eixos temáticos, envolvendo os temas geradores Nanociência e Nanotecnologia, à luz do enfoque de CTSA.

\section{Resultados e discussão}

A busca empreendida, até 31 de março de 2010, permitiu a obtenção de 24 artigos no SCIELO e 257 artigos no PUBMED. O conjunto de textos (artigos, capítulos de livros e livros) foi lido, organizando-se as observações coligidas em torno dos possíveis enfoques para o desenvolvimento de atividades didáticas, no bojo da ECT, a partir da perspectiva de CTSA.

A nanotecnologia foi eleita por ser uma temática atual, presente constantemente nos diferentes tipos de mídia - possibilitando, assim, a contextualização da ciência e da tecnologia, com as demandas atuais da sociedade - além de se constituir como saber essencialmente interdisciplinar (ARAKI, 2007; TEDESCO, SIMIONI e PRIMO, 2007). O termo - constituído por três radicais de origem grega: nano significa anão; tecno diz respeito à arte (técnica); e logia refere-se a discurso (saber ou ciência) - engloba um conjunto de técnicas empregadas para manipular a matéria na escala de átomos e moléculas, envolvendo partículas extremamente reduzidas, da ordem da bilionésima parte do metro - à nanoescala pertence tudo que se apresenta um tamanho característico de 0,1 a 100 nanômetros; $1 \mathrm{~nm}=0,000000001 \mathrm{~m}=1 \times 10^{-9} \mathrm{~m}-$ , mil vezes menor que o diâmetro da hemácia, uma célula sanguínea (BHUSHAN, 2003). Este campo do saber nasce na segunda metade do século XX, quando o físico Richard Feynman Prêmio Nobel de Física de 1965 -, ao proferir uma palestra em 1959, comenta, explicitamente, sobre a existência de "mais espaço lá embaixo", em uma clara referência à possibilidade de manipulação do mundo em nível atômico (FEYNMAN, 2009). Posteriormente, em 1981, foi dado um passo importante neste sentido: a criação, pela IBM de Zurich, da Scanning tunneling microscope (STM - Microscopia de varredura por tunelamento), tornando-se possível a observação e a manipulação da matéria com resolução atômica (WEISSMÜLLER, CIDADE e BISCH, 2007). De fato,

Estamos no limiar de uma verdadeira revolução tecnológica, cuja evolução deverá abranger décadas, com um impacto que provavelmente deverá superar o de todas as revoluções técnicas do passado. Dela resultarão materiais inéditos, grandes avanços na medicina e na farmacologia, métodos muito mais eficientes para a indústria química e petroquímica, computadores com um grau de sofisticação e complexidade sem precedentes - provavelmente baseados em outros princípios físicos -, maior eficiência no uso de energia, grandes inovações na área do meio ambiente e vários outros avanços que podemos apenas vislumbrar. (VALADARES, CHAVES e ALVES, 2005, p. 51-52) 
Ao investigar - e manipular - a matéria neste nível de grandeza, a nanotecnologia e a nanociência têm a possibilidade de produzir artefatos e técnicas capazes de interferir em diferentes sistemas materiais, algo que se tem observado hodiernamente. Desta feita, para que a compreensão de seus meandros - pressupostos e consequências - possa ser maximizada, o ideal é que as diferentes vertentes do saber-fazer possam ser agregadas, constituindo eixos temáticos, os quais funcionariam como núcleos conceituais capazes de ser compreensíveis pelos discentes. Assim, pois, utilizando-se as características dos conteúdos científicos específicos - obtidos a partir da leitura de textos publicados nos últimos anos (especialmente BHUSHAN, 2003; QUINA, 2004; BORSCHIVER et al., 2005; KUBIK, BOGUNIA-KUBIK e SUGISAKA, 2005; PIMENTEL et al., 2007; TEDESCO, SIMIONI e PRIMO, 2007; WEISSMÜLLER, CIDADE e BISCH, 2007; FERREIRA e RANGEL, 2009) -, foram estabelecidos quatro eixos temáticos para a abordagem na ECT: (1) nanotecnologia e nanomateriais, (2) nanobiotecnologia e saúde, (3) nanotecnologia e meio ambiente, e (4) nanotecnologia, ética e política.

\section{Nanotecnologia e nanomateriais}

A pesquisa dirigida ao desenvolvimento de nanomateriais tem por objetivo "controlar com precisão a morfologia em dimensão nanométrica das substâncias ou partículas para produzir materiais nanoestruturados" (ARAKI, 2007; TEDESCO, SIMIONI e PRIMO, 2007). Nesta esfera, a nanotecnologia

[...] estende a ciência de materiais para o domínio de partículas e interfaces com dimensões extremamente pequenas, da ordem de um a cem nanômetros. Partículas deste tamanho, ou "nanopartículas", apresentam uma grande área superficial e, freqüentemente, exibem propriedades mecânicas, ópticas, magnéticas ou químicas distintas de partículas e superfícies macroscópicas. O aproveitamento dessas propriedades em aplicações tecnológicas forma a base da nanotecnologia de materiais. Há, também, uma área ainda incipiente da nanotecnologia, denominada nanotecnologia molecular ou nano-fabricação ("nanomanufacturing"), que almeja o desenvolvimento de sistemas nanométricos autoreplicantes (nano-robôs ou "nano-bots") capazes de fabricar, sob medida, materiais ou objetos através da manipulação da matéria a nível molecular. (QUINA, 2004, p. 1028)

É importante mencionar que o nível de intervenção da nanotecnologia explicita características físicas e químicas - p. ex., condutividade, reatividade, temperatura de fusão, e outros - bastante diferentes dos materiais de origem (VALADARES, CHAVES e ALVES, 2005; LÊDO, 2006; FERREIRA e RANGEL, 2009). Estas díspares propriedades - por exemplo, o ouro é habitualmente amarelo... o "nano-ouro", vermelho... - podem concorrer para a identificação de novos materiais com propriedades extremamente úteis em termos de emprego tecnológico - pode-se mencionar que o $\mathrm{CaCO}_{3}$ (carbonato de cálcio, giz, significativamente 
Siqueira-Batista, R. et al.

frágil) pode ser mais duro do que o aço em escala nanométrica. Atividades pedagógicas interdisciplinares - envolvendo biologia, física, química e outros saberes -, podem ser desenhadas à luz da abordagem desta temática (BORSCHIVER et al., 2005).

\section{Nanobiotecnologia e saúde}

As possibilidades neste domínio - síntese da nanoengenharia com a manipulação de sistemas biológicos - são amplas, incluindo (KUBIK, BOGUNIA-KUBIK e SUGISAKA, 2005; VALADARES, CHAVES e ALVES, 2005; PATEL et al., 2006; PIRES-DO-PRADO et al., 2006; ROSSI-BERGMANN e FRÉZARD, 2007; SILVA e FACCIOLI, 2007; SIQUEIRABATISTA et al., 2008):

(a) 'Fabricação' de nanobiomateriais, área de interseção com o campo de investigação dos nanomateriais;

(b) Desenvolvimento de exames diagnósticos em nanoescala, como nanorastreadores de agentes infecciosos;

(c) Implementação de modalidades terapêuticas, com destaque para o nanoensapsulamento de fármacos - já em uso, por exemplo, para moléstias infecciosas por fungos e protozoários , para o emprego de nanopartículas magnéticas destinadas ao tratamento do câncer - guiadas à região do tumor pelo uso de ímãs - e para a criação (futura) de nanodispositivos "inteligentes" / nanorobots - também chamados nanobots -, dentre outros;

(d) Produção de métodos de prevenção, por meio do desenvolvimento de imunobiológicos - especialmente vacinas -, já estando em investigação imunoprofilaxia para leishmaniose e tuberculose.

As aplicações relacionadas à nutrição humana são também bastante diversificadas. $\mathrm{O}$ emprego de nanopartículas como antioxidantes, de sistemas "inteligentes" para a absorção de ingredientes funcionais e de embalagens contendo nanossensores para detecção de microrganismos e/ou toxinas estão entre as fronteiras mais promissoras no âmbito da ciência da nutrição (WEISS, TAKHISTOV E CLEMENTS, 2006; CHUN, 2009).

Outras aplicações possíveis, também descritas, incluem a preparação de nanocosméticos, a realização de nanocirurgias, as perspectivas em termos de neuroproteção e de regeneração neural, boa parte das quais se referindo, ainda, a usos vindouros. Neste eixo temático, é igualmente produtiva a implementação de atividades interdisciplinares, envolvendo as ciências da natureza, da saúde e as humanidades.

\section{Nanotecnologia e meio ambiente}

As investigações sobre as possíveis influências do uso de nanopartículas no ambiente são ainda recentes, não havendo definição clara a respeito da sua utilização e regulamentação, ponderando-se sobre os eventuais benefícios (QUINA, 2004; VALADARES, CHAVES e ALVES, 2005; LÊDO, 2006; SURENDIRAN et al., 2009; WANG et al., 2010):

(a) Detecção e acompanhamento da polvição, por meio da preparação de nanosensores, mais sensíveis e mais específicos, para a detecção e o monitoramento ambiental de poluentes - 
orgânicos e inorgânicos -, com aplicabilidade, por exemplo, na avaliação dos níveis de poluentes em alimentos e em outros produtos de consumo humano;

(b) Tratamento da poluição, em decorrência da relevante propriedade de adsorção de diferentes substâncias - por exemplo, metais e compostos orgânicos -, pela grande área superficial das nanopartículas; tem sido descrito, igualmente, o emprego de nanopartículas magnéticas e a utilização de nanotubos de carbono para a dessalinização da água; ademais, é pensável, a longo prazo, que nano-bots sejam capazes de agir na descontaminação ambiental;

(c) Profilaxia dos agravos relacionados à poluição, destacando-se o emprego de nanomateriais catalíticos - os quais amplificam a eficiência e a seletividade de processos industriais -, concorrendo para um maior aproveitamento de matérias-primas, com reduzido dispêndio de energia e menor produção de resíduos indesejáveis.

Ainda que se possa conjecturar sobre estes promissores usos, as características físicoquímicas das nanopartículas facilitam sua dispersão - na atmosfera, nas águas, nos solos e nos sistemas vivos - e, igualmente, dificultam sua remoção por técnicas habituais de filtração, tendo tais ocorrências efeitos imprevisíveis - de fato, há observações recentes da presença de nanopartículas no fígado de animais usados em pesquisas, as quais podem "ganhar" a cadeia alimentar (SBPC/Labjor, 2002). Com efeito, a discussão segue envolta em grande controvérsia, aspecto que pode ser explorado em atividades interdisciplinares de ensino de ciências - formulando estratégias pedagógicas para trabalhar o tema em sala de aula -, a partir da realização de debates sobre os prós e os contras do uso dessa tecnologia, enfocando os aspectos ambientais.

\section{Nanotecnologia, ética e política}

Os problemas éticos e políticos atinentes à nanociência e à nanotecnologia podem ser vistos como sínteses dos três eixos temáticos anteriores. Assim, pois, as perguntas envolvendo a produção de nanomateriais, a aplicação à saúde e o impacto ambiental da nanotecnologia, têm como pano de fundo as consequências - boas ou más - sobre o planeta (SHINN e MARCOVICH, 2009). Assim:

[...] as potencialidades da nanotecnologia são ao mesmo tempo entusiasmantes e amedrontadoras. Por um lado, o desenvolvimento de supercomputadores, de novos medicamentos, de novos materiais para uso na astronáutica, na reciclagem, num sem-número de indústrias inéditas. Por outro, a construção de armas apavorantes - diminutos invasores invisíveis, do tamanho de uma bactéria ou mesmo de um vírus, programados por alguma potência inimiga ou por um demente, poderiam penetrar numa dada região transportados por uma rajada de vento e transformar os ossos da população inteira numa pasta pegajosa. (OLIVEIRA, 2002, p. 211)

Estas indagações podem ser apreciadas pelos estudantes da área científica e tecnológica, compondo, de modo interessante, os conceitos das ciências da natureza - trabalhados interdisciplinarmente pela própria natureza da nanotecnologia - com a argumentação instada 
Siqueira-Batista, R. et al.

pelas ciências humanas, em termos de ética e política (SCHULTE e SALAMANCA-BUENTELLO, 2007).

A partir desses enfoques, pretende-se estimular as conversações sobre conteúdos específicos de díspares disciplinas - Biologia, Filosofia, Física, História, Química e Sociologia, dentre outras -, por meio da discussão de um tema atual, provocando, no discente, o interesse pela construção de conceitos - capazes de contribuir para o debate -, além de permitir uma contextualização sobre os conteúdos específicos aprendidos na escola, com as distintas temáticas em pauta no cenário contemporâneo. Assim, propõe-se que o educando estará mais estimulado para participar das diferentes atividades propostas pelos docentes - independentemente da metodologia de ensino-aprendizagem adotada -, pois perceberá, de forma clara, como seu aprendizado escolar poderá ajudá-lo a melhor compreender a realidade.

\section{Considerações finais}

A nanociência e a nanotecnologia são campos em pleno desenvolvimento, com promissoras expectativas em múltiplos aspectos do mundo contemporâneo:

[...] é de se supor que a nanotecnologia afetará drasticamente o modo como vivemos e vemos o mundo. O meio ambiente, o funcionamento de nosso corpo e de outros organismos vivos e a busca de uma matriz energética inédita serão alvos dos futuros avanços, gerando grandes oportunidades e desafios. (VALADARES, CHAVES e ALVES, 2005, p. 69-70)

Os reflexos do nanoconhecimento na existência das pessoas, das coletividades, da civilização e, quiçá, na própria biosfera são, evidentemente, de difícil mensuração neste momento, aumentando a responsabilidade da comunidade científica e das instituições de ensino - atores que devem lançar mão de todos os esforços para divulgar informações corretas sobre nanotecnologia. Neste particular, vale lembrar o compromisso proposto por Chassot:

A nossa responsabilidade maior no ensinar Ciência é procurar que nossos alunos e alunas se transformem, com o ensino que fazemos, em homens e mulheres mais críticos. Sonhamos que, com nosso fazer Educação, os estudantes possam tornar-se agentes de transformação para melhor - do mundo em que vivemos. (CHASSOT, 2006, p. 31)

De acordo com esta perspectiva, muito mais do que um domínio meramente tecnocientífico, a condução de pesquisas envolvendo nanociência e nanotecnologia deve ser ampliada ao questionamento da sociedade civil - tornando-se, definitivamente, uma questão ético-política -, de modo que decisões melhor pensadas e responsáveis possam ser tomadas. A ECT crítica é extremamente importante neste mister - especialmente ao se empregar o enfoque de CTSA -, por criar espaços democráticos para o debate em sala de aula, concorrendo para a formação de cidadãos mais responsáveis com o próximo, com a sociedade e com o meio ambiente. 
Nanociência e nanotecnologia como temáticas ...

\section{Referências}

ALBUQUERQUE, V. S.; SIQUEIRA-BATISTA, R.; TANJI, S.; MOÇO, E. T.-S. M. Currículos disciplinares na área de saúde: ensaio sobre saber e poder. Interface Comunicação, Saúde e Educação, Botucatu, v. 13, n. 31, p. 261-272, 2009.

AMORIM, A. C. R. O que foge do olhar das reformas curriculares: nas aulas de biologia, o professor como escritor das relações entre ciência, tecnologia e sociedade. Ciência e Educação, Bauru, v. 7, n. 1, p. 47-65, 2001.

ARAKI, K. Estratégia supramolecular para a nanotecnologia. Química Nova, São Paulo, v. 30, n. 6 , p. $1484-1490,2007$.

BOMFIM, A. M. Educação ambiental crítica: para além do positivismo e aquém da metafísica. In: ARAÚJO, F. M. B; ROÇAS, G.; RODRIGUES, D. C. P. (Orgs.). Energia para um mundo eco-lógico e saudável. 1. ed. Nilópolis: IFRJ, 2009. v. 1. p. 9-18.

BOMFIM, A. M.; SIQUEIRA-BATISTA, R. Conversações entre as ciências humanas e sociais e as ciências da natureza: um ensaio sobre a práxis docente em cursos de licenciatura em física, química e matemática. In: ENCONTRO NACIONAL DE PESQUISA EM EDUCAÇ̃̃O EM CIÊNCIAS, 7., 2009, Florianópolis. Anais... Florianópolis: Abrapec, 2009. 1 cd-rom.

BORSCHIVER, S.; GUIMARÃES, M. J. O. C.; SANTOS, T. N.; SILVA, F. C.; BRUM, P. R. C. Patenteamento em nanotecnologia: estudo do setor de materiais poliméricos nanoestruturados. Polímeros, São Carlos, v. 15, n. 4, p. 245-248, 2005.

BHUSHAN, B. Springer handbook of nanotechnology. Ohuio: Springer-Verlag, 2003.

CHASSOT, A. Alfabetização científica: questões e desafios para a educação. 4. ed. Ijuí: Unijuí, 2006.

CHUN, A. L. Will the public swallow nanofood? Nature Nanotechnology, London, v. 4, n. 12 , p. $790-1,2009$.

FARIAS, C. R. O.; CARVALHO, W. L. P. Desvelando relações ciência-tecnologia-sociedadeambiente a partir de um processo judicial sobre danos ambientais. Revista Eletrônica do Mestrado em Educação Ambiental, Rio Grande, v. 17, n. 2, p. 316-330, 2006.

FERREIRA, H. S.; RANGEL, M. C. Nanotecnologia: aspectos gerais e potencial de aplicação em catálise. Química Nova, São Paulo, v. 32, n. 7, p. 1860-1870, 2009.

FEYNMAN, R. There's plenty of room at the bottom, 1959. Disponível em: <http:// www.its.caltech.edu/ feynman/plenty.html>. Acesso em: 20 nov. 2009.

FOUREZ, G. Crise no ensino de ciências? Investigações em Ensino de Ciências, Porto Alegre, v. 8, n. 2, p. 1-14, 2003.

GEWIN, V. Big opportunities in a small world. Nature, London, v. 460, n. 7254, p. $540-541,2009$. 
Siqueira-Batista, R. et al.

KUBIK, T.; BOGUNIA-KUBIK, K.; SUGISAKA, M. Nanotechnology on duty in medical applications. Current Pharmaceutical Biotechnology, Dallas-Fort Worth, v. 6, n. 1, p. 17-33, 2005.

LÊDO, J. C. S. Questões bioéticas suscitadas pela nanotecnologia. 2006. 120 f.

Dissertação (Mestrado em Bioética) - Centro Universitário São Camilo, São Paulo, 2006.

MOREIRA, I. C. A inclusão social e a popularização da ciência e tecnologia no Brasil. Inclusão Social, Brasília, v. 1, n. 2, p. 11-16, 2006.

MORTIMER, E. F. Uma agenda para a pesquisa em educação em ciências. Revista Brasileira de Pesquisa em Educação em Ciências, Belo Horizonte, v. 2, n. 1, p. 25-35, 2002.

MUENCHEN, C.; AULER, D. Configurações curriculares mediante o enfoque CTS: desafios a serem enfrentados na educação de jovens e adultos. Ciência e Educação, Bauru, v. 13, n. 3, p. 421-434, 2007.

OLIVEIRA, L. A. Valores deslizantes: esboço de um ensaio sobre técnica e poder. In: NOVAES, A. (Org.). O avesso da liberdade. São Paulo: Companhia das Letras, 2002. p. 507-519.

PATEL, G. M.; PATEL, G. C.; PATEL, R. B.; PATEL, J. K.; PATEL, M. Nanorobot: a versatile tool in nanomedicine. Journal of Drug Targeting, Safat, v. 14, n. 2, p. 63-67, 2006.

PIMENTEL, L. F.; JACOME JUNIOR, A. T.; MOSQUEIRA, V. C. F.; SANTOSMAGALHAES, N. S. Nanotecnologia farmacêutica aplicada ao tratamento da malária. Revista Brasileira de Ciências Farmacêuticas, São Paulo, v. 43, n. 4, p. 503-514, 2007.

PIRES-DO-PRADO, H. J.; GOMES, A. P.; BRAZIELLAS, D.; ESPERIDIÃO ANTONIO, V.; GELLER, M.; MORGADO, F.; HELAYËL-NETO, J. A.; SIQUEIRA-BATISTA, R. Nanoimunologia: perspectivas atuais e importância na formação médica. Revista Brasileira de Educação Médica, Gramado, v. 30, p. 528, 2006.

PUBMED. U. S. National Library of Medicine. Disponível em: <http:// www.ncbi.nlm.nih.gov/pubmed/>. Acesso em: 31 mar. 2010.

QUINA, F. H. Nanotecnologia e o meio ambiente: perspectivas e riscos. Química Nova, São Paulo, v. 27, n. 6, p. 1028-1029, 2004.

RÔÇAS, G.; MONTEIRO, J. A.; SIQUEIRA-BATISTA, R. Transdisciplinaridade no ensino de ciências: debates teóricos em torno do conceito de espécie. Revista Ciência em Tela, Rio de Janeiro, v. 1, n. 2, p. 1-9, 2008.

ROSSI-BERGMANN, R.; FRÉZARD, F. Aplicação da nanotecnologia para tratamento e vacinação da leishmaniose. In: MORALES, M. M. Terapias avançadas: células-tronco, terapia gênica e nanotecnologia aplicada à saúde. São Paulo: Atheneu, 2007. p. 265-277. 
Nanociência e nanotecnologia como temáticas ...

ROUSSEAU, J. J. Discours sur les sciences et les arts: oeuvres complètes. Paris: Seuil, 1971.

SANT'ANNA, I. M.; MENEGOLLA, M. Didática: aprender a ensinar. 8. ed. São Paulo: Loyola, 2007.

SANTOS, B. S. Um discurso sobre as ciências. São Paulo: Cortez, 2003.

SBPC/LABJOR. Sociedade Brasileira para o Progresso da Ciência - Laboratório de Estudos Avançados em Jornalismo. Vantagens e riscos da nanotecnologia ao meio ambiente.

São Paulo, 2002. Disponível em: <http://www.comciencia.br/reportagens/nanotecnologia/ nano04.htm>. Acesso em: 2 fev. 2010.

SCIELO. Scientific Electronic Library Online. Disponível em: < http://www.scielo.br> Acesso em: 31 mar. 2010.

SCHULTE, P. A.; SALAMANCA-BUENTELLO, F. Ethical and scientific issues of nanotechnology in the workplace. Ciência e Saúde Coletiva, Rio de Janeiro, v. 12, n. 5, p. 1319-1332, 2007.

SHINN, T.; MARCOVICH, A. Padrões sociointelectuais da pesquisa em nanoescala: laureados com o Prêmio Feynman de Nanotecnologia, 1993-2007. Scientia e Studia, São Paulo, v. 7, n. 1, p. 11-39, 2009.

SILVA, C. L.; FACCIOLI, L. H. DNA microparticulado como vacina contra câncer e tuberculose. In: MORALES, M. M. (Org.). Terapias avançadas: células-tronco, terapia gênica e nanotecnologia aplicada à saúde. São Paulo: Atheneu, 2007. p. 279-288.

SIQUEIRA-BATISTA, R.; MARIA DA SILVA, L.; MACEDO JÚNIOR, M. A. V.; ALVES FERREIRA, R.; PIRES-DO-PRADO, H. J.; SIQUEIRA-BATISTA, R.; HELAYËL-NETO, J. A. Nanociência e nanotecnologia: aplicações em medicina In: ENCONTRO NACIONAL DE FÍSICA DA MATÉRIA CONDENSADA, 31., 2008, Águas de Lindóia. Anais... São Paulo: Sociedade Brasileira de Fìsica, 2008. 1 cd-rom.

SIQUEIRA-BATISTA, R.; SIQUEIRA-BATISTA, R. Os anéis da serpente: a aprendizagem baseada em problemas e as sociedades de controle. Ciência e Saúde Coletiva, Rio de Janeiro, v. 14, n. 4, p. 1183-1192, 2009.

SIQUEIRA-BATISTA, R.; MEDEIROS-SOUZA, R. R.; MARIA DA SILVA, L.; SILVA, C. A.; PIRES-DO-PRADO, H. J.; RÔÇAS, G. Nanotecnologia e ensino de ciências à luz do enfoque CTS: uma viagem a Lilliput. Ciências e Idéias, Nilópolis, v. 1, n. 1, p. 76-86, 2009.

SURENDIRAN, A.; SANDHIYA S.; PRADHAN S.C.; ADITHAN C. Novel applications of nanotechnology in medicine. Indian Journal of Medical Research, New Delhi, v. 130, n. 6, p. 689-701, 2009.

TEDESCO, A. C.; SIMIONI, A. R.; PRIMO, F. L. Introdução à nanotecnologia. In: MORALES, M. M. (Org.). Terapias avançadas: células-tronco, terapia gênica e nanotecnologia aplicada à saúde. São Paulo: Atheneu, 2007. p. 237-246. 
Siqueira-Batista, R. et al.

TEIXEIRA, P. M. M. A educação científica sob a perspectiva da pedagogia histórico-crítica e do movimento CTS no ensino de ciências. Ciência e Educação, Bauru, v. 9, n. 2, p. $177-190,2003$.

TOMA, H. E. Interfaces e organização da pesquisa no Brasil: da Química à Nanotecnologia. Química Nova, São Paulo, v. 28, suplemento, p.S48-S51, 2005.

VALADARES, E. C.; CHAVES, A.; ALVES, E. G. Aplicações da física quântica: do transistor à nanotecnologia. São Paulo: Livraria da Física, 2005.

WANG, H.; WANG, Z.; SONG, D.; WANG, J.; GAO, J.; WANG, L.; KONG, D.; YANG, Z. Enzyme-assisted formation of nanosphere: a potential carrier for hydrophobic compounds. Nanotechnology, Bristol, v. 21, n. 15, p. 155602, 2010.

WEISS, J.; TAKHISTOV, P.; CLEMENTS, J. C. Functional materials in food nanotechnology. Journal of Food Science, West Sussex, v. 71, n. 9, p. R107-R116, 2006.

WEISSMÜLLER, G.; CIDADE, G. A. G.; BISCH, P. M. Microscopia de força atômica: nanoscopia, nanomanipulação e nanocaracterização de biomateriais. In: MORALES, M. M. (Org.). Terapias avançadas: células-tronco, terapia gênica e nanotecnologia aplicada à saúde. São Paulo: Atheneu, 2007. p. 307-321.

Artigo recebido em maio de 2010 e aceito em julho de 2010. 